\title{
Dynamic Response of Ground Supported Rectangular Water Tanks to Earthquake Excitation
}

\author{
Birhane Aregawi* and Abdulaziz Kassahun \\ School of Civil Engineering, Ethiopian Institute of Technology-Mekelle (EiT-M), Mekelle \\ University, Mekelle, Ethiopia (*birhanea.sec@mu.edu.et).
}

\begin{abstract}
This study investigates an idealized ground supported reinforced concrete rectangular water tank under earthquake excitation. A linear three-dimensional finite element analysis and SAP2000 software have been used to predict tank response. The variable analysis parameters considered are the aspect ratio (tank height to length ratio) and tank water level, while the tank wall thickness is taken as a constant. The convective and impulsive masses are also represented by spring-mass model in the time history analysis for El-Centro earthquake ground motion record. Five tank models with a capacity of 216, 288, 360, 432 and $504 \mathrm{~m}^{3}$ were developed and analyzed for hydrodynamic and hydrostatic effects. In general the results show that, there is a smooth increase in the moment and displacement of both hydrostatic and hydrodynamic analysis with a decrease in aspect ratio. The top displacement and moment for the hydrodynamic effects are greater than the hydrostatic results and it is observed that the maximum hydrodynamic moment is $91.3 \%$ higher than the corresponding maximum hydrostatic moment. Likewise the displacement obtained from hydrodynamic analysis is $63.58 \%$ more than the corresponding hydrostatic value.
\end{abstract}

Keywords: Hydrodynamic effect, Convective and Impulsive, Rectangular tanks, Finite Element Models, Time history, SAP2000, Ethiopia.

\section{INTRODUCTION}

Storage tanks are used for storing water, inflammable liquids toxic materials and other petrochemicals. Tanks constructed of reinforced concrete are most popular and widely used components in any major water distribution network for public utility and industrial facilities. Thus, it is evident that such tanks should be designed to withstand lateral loads in addition to vertical loads. However, due to lack of seismic load consideration; damages of storage tanks in recent earthquakes have been observed and extensively studied by different researchers to render its vitality (Housner, 1963). Housner was the first who considered the hydrodynamic pressure distribution developed in rigid tanks for a horizontal base excitation (Jaiswal and Jain, 2005a,b). In fact, prior to the advent of modern computers and the widespread of numerical methods in structural engineering, water storage tanks were analyzed mathematically using closed form shell analysis solutions together with some relevant design curves. However, those approaches were 
conventionally limited to hydrostatic effects.

Thus, using numerical methods for the rigorous evaluation of the dynamically induced stresses on the tank walls is found to be accurate approach as it involves the interaction between the lateral displacement of the tank and that of the fluid motion.

This dynamic interaction due to lateral movement affects the strength and behavior of the tank. Hence, there is a need to understand the behavior of liquid retaining tanks and to consider the latest advances in the design so that they are not vulnerable under earthquake loads. In this study, behavior of a typical ground supported tank under seismic load has been studied using the finite element model.

\section{DYNAMIC MODELING FOR SEISMIC ANALYSIS}

\subsection{Review of design Codes}

Some of the structural design codes that tackle fluid tank systems are the American Concrete Institute, ACI 350.3, the Euro Code 8 and the Standards Association of New Zealand, NZS. These codes address ground supported circular and rectangular concrete tanks having fixed or flexible bases. This condition is relevant to this study; the aim of which is to create an idealized model suitable for representing the vibrating fluid tank system by a proper spring-mass system which considerably simplifies the interaction. Proper seismic analysis accounts for the inertia forces of the accelerating structure as well as the inertia forces of the accelerating fluid which the tank contains. The ACI uses the mechanical model of Housner but with the generalizations introduced by Wozniak and Mitchel (ACI350.3, 2001).

Euro Code 8 mentions the acceptable procedure of Valestos and Yang for modeling rigid water tanks and the guidelines of NZS are essentially similar to the Euro Code 8 but introduce a reduction factor for the mass of the tank wall in order to compensate for the conservatism in evaluating the impulsive force. Furthermore, when dealing with a rectangular tank it suggests using half of the width of the rectangular tank as a radius of an equivalent circular cylindrical tank (Eurocode 8, 1998). In regard to the combination procedure of the impulsive forces and the convective forces the ACI and NZS suggest the Square Root Sum of the Squares (SRSS) rule; while the Euro Code suggests the absolute sum (ABSSUM) combination rule (Helou, 2014). 


\subsection{Spring - Mass Model}

Dynamic analysis of liquid storage tank is a complex problem involving fluid-structure interaction. Based on numerous analytical, numerical and experimental studies, a simple spring mass models of tank-liquid system have been developed to evaluate hydrodynamic effects (Jaiswal and Jain, 2005a,b).

When a tank containing liquid with a free surface is subjected to horizontal earthquake ground motion, tank wall and liquid are subjected to horizontal acceleration. The liquid in the lower region of tank behaves like a mass that is rigidly connected to tank wall. This mass is termed as impulsive liquid mass, which accelerates along with the wall and induces impulsive hydrodynamic pressure on tank wall and similarly on the base. Liquid mass in the upper region of tank undergoes sloshing motion. This mass is termed as convective liquid mass and it exerts convective hydrodynamic pressure on tank wall and base. Thus, total liquid mass gets divided into two parts, i.e., impulsive mass and convective mass. In spring mass model of tank-liquid system, these two liquid masses are to be suitably represented. A qualitative description of impulsive and convective hydrodynamic pressure distribution on tank wall and base is given in figure 1 .

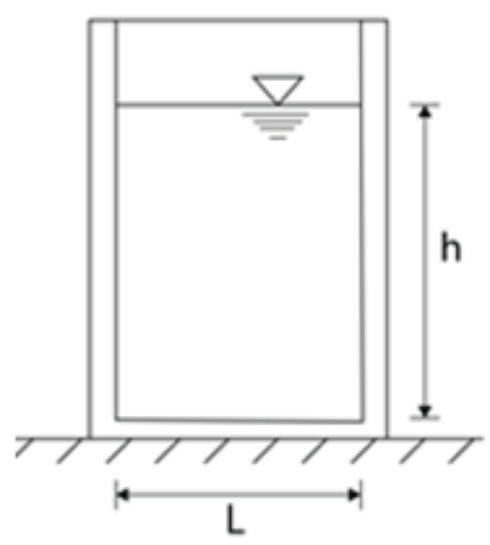

(a) Tank

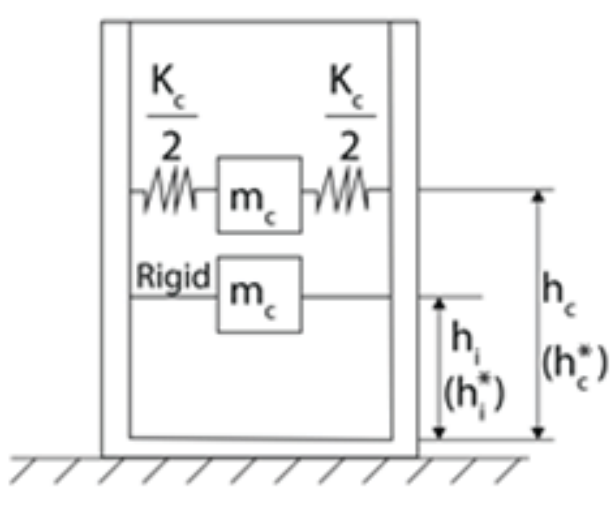

(b) Spring Mass Model

Figure 1. Spring-Mass Model for Ground Supported Rectangular Tank (Jaiswal and Jain, 2005).

The parameters of spring mass model depend on tank geometry and are originally proposed by Housner (1963). The parameters shown in table 1 are slightly different from those given by 
Housner (1963) and are taken from American Concrete Institute (ACI350.3, 2001).

Table 1. Expressions for parameters of spring mass model.

\begin{tabular}{|l|l|}
\hline$\frac{m_{i}}{m}=\frac{\tanh (0.866 L / h)}{0.866 L / h}$ & $\frac{m_{c}}{m}=\frac{\tanh (0.866 L / h)}{0.866 L / h}$ \\
\hline$\frac{h_{i}}{h}=0.375$ for $h / L \leq 0.75$ & $\frac{h_{i}}{h}=0.5-\frac{0.09375}{h / L} \quad$ for $h / L>0.75$ \\
\hline$\frac{h_{i}^{*}}{h}=\frac{0.866 L / h}{2 \tanh (0.866 L / h)}-0.125$ for $h / L \leq 1.33$ & $\frac{h_{i}^{*}}{h}=0.45$ for $h / L>1.33$ \\
\hline$\frac{h_{c}}{h}=1-\frac{\cosh \left(3.16 \frac{h}{L}\right)-1.0}{3.16 \frac{h}{L} \sinh \left(3.16 \frac{h}{L}\right)}$ & $\frac{h_{c}^{*}}{h}=1-\frac{\cosh \left(3.16 \frac{h}{L}\right)-2.01}{3.16 \frac{h}{L} \sinh \left(3.16 \frac{h}{L}\right)}$ \\
\hline$K_{c}=0.833 \frac{m g}{h} \tanh ^{2}\left(3.16 \frac{h}{L}\right)$ & Note; Notations are defined in figure 1 \\
\hline
\end{tabular}

\section{DESCRIPTION OF THE TANK STRUCTURE}

The water tank structures analyzed in the present study are a ground supported rectangular storage tanks with a volume of 216, 288, 360, 432 and $504 \mathrm{~m}^{3}$. The geometries are selected taking into account the limitation of rectangular shaped tanks for containing higher volume due to lack of uniform stress distribution that leads to local failure. The contained liquid is assumed to be water with a density of $10 \mathrm{kN} / \mathrm{m}^{3}$. The planar dimensions of the tanks are $6 \mathrm{~m} \mathrm{x} 6 \mathrm{~m}, 8 \mathrm{~m} \mathrm{x}$ $6 \mathrm{~m}, 10 \mathrm{~m} \times 6 \mathrm{~m}, 12 \mathrm{~m} \times 6 \mathrm{~m}$ and $14 \mathrm{~m} \times 6 \mathrm{~m}$ with a height $(\mathrm{h})$ of $6 \mathrm{~m}$ and the values of the aspect ratio(A) i.e. the ratio of the length to height of the tank $(1 / h)$, is used for analysis and comparison of results. The tank structure is idealized to be of $0.3 \mathrm{~m}$ thickness reinforced concrete wall system with a concrete grade of C-25. A damping coefficient of $0.5 \%$ is used for the analysis of the first and second modes while $5 \%$ is adopted for other modes.

\section{SEISMIC GROUND MOTION}

El-Centro seismic ground motion, represented by acceleration with duration of $11.98 \mathrm{sec}$ and peak ground acceleration (PGA) of $0.3194 \mathrm{~g}$, is adopted to simulate the dynamic effect of the tank structures. The tanks have been analyzed in the critical direction and the first fifteen seconds 
of time period were considered for analyzing the tanks.

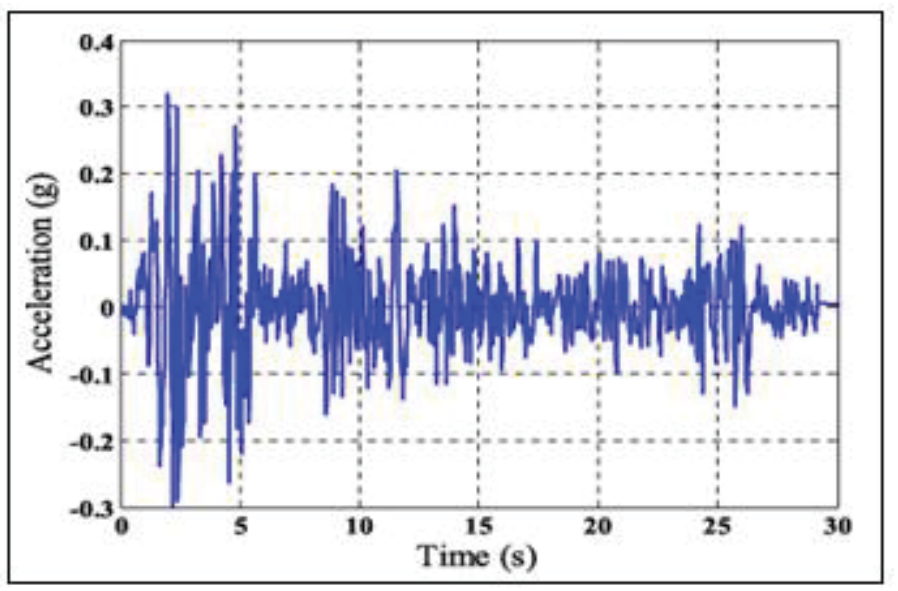

Figure 2. Accelerogram record of El Centro earthquake, 18-May-1940 (Chopra, 2006).

\section{FEM MODELING AND DETAILED DYNAMIC ANALYSIS}

The numerical analysis of the rectangular storage tank structures is performed on the basis of detailed FE model implemented with the help of the available Finite Element routines in SAP2000 software package. The wall thickness is approximated to be uniform thickness instead of tapered wall and the base slab modeling is not considered for simplicity.

Furthermore, the following assumptions are made to simplify the dynamic analysis procedure;

(1) The material of the tank is linearly elastic, isotropic and homogeneous;

(2) The contained water is with zero viscosity; and

(3) The base is connected rigidly to the tank wall.

Impulsive and convective masses are added as joint masses at the junction of the springs which are connected to the walls at different levels. A higher value of spring stiffness is used to depict the rigid characteristics of the impulsive mass. The FEM rectangular tank model is numerically analyzed by means of a full transient linear analysis. The governing equations of motion can be expressed in matrix form as shown in equation 1 (Chopra, 2006). The seismic effect in the direction perpendicular to the long wall is considered and equations of dynamic equilibrium are solved using modal-time history analysis.

$[M]\{\ddot{y}\}+[C]\{\dot{y}\}+[k]=-[M]\{R\} \ddot{u}_{g}$ 
With $[\mathrm{M}],[\mathrm{C}]$ and $[\mathrm{K}]$ being the modal mass, modal damping and modal stiffness matrices of the tank structure respectively. $\{R\}$ is an influence coefficient matrix and $\ddot{u}_{\mathrm{g}}$ the ground acceleration. The analysis is performed for 20 modes to enable $89.0 \%$ of modal mass participation. The typical 3D view of Finite Element model is shown in figure 3 and plans at convective and impulsive heights of the FE model is shown in figure 4.

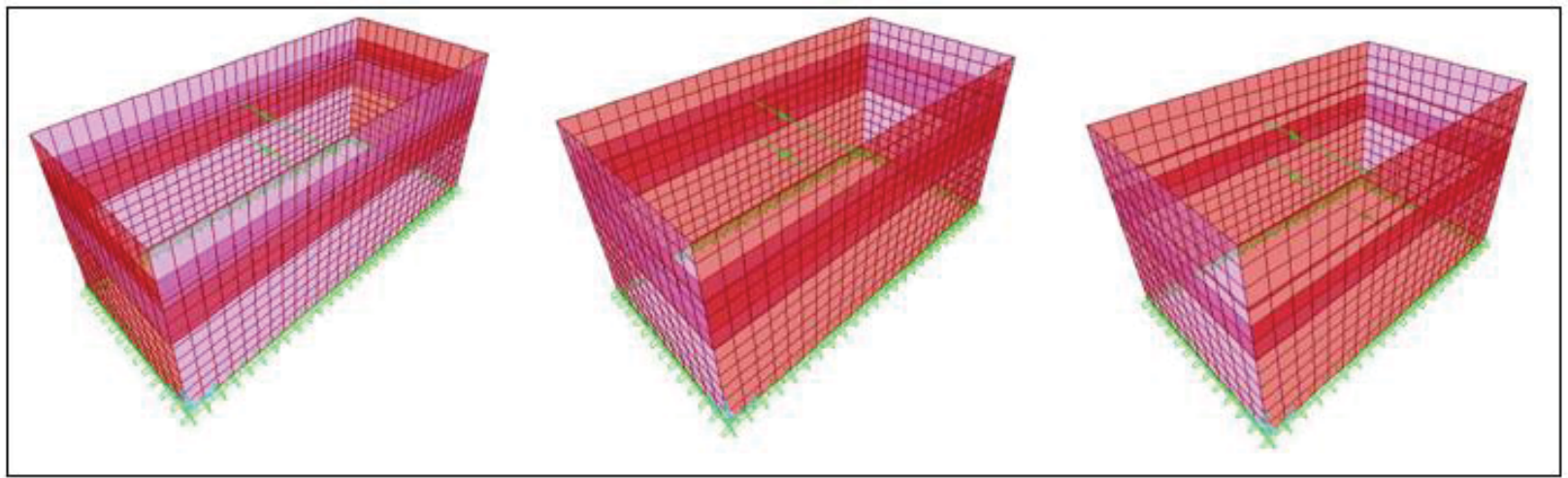

Figure 3. 3D- analytical models for different volumes of rectangular tanks.

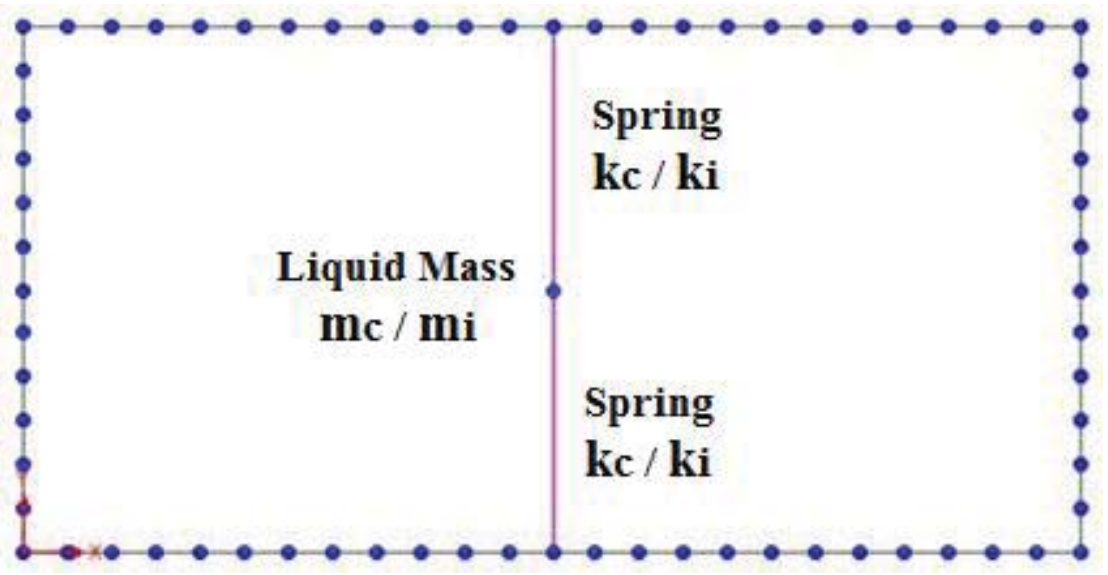

Figure 4. Impulsive and Convective Mass-Spring Interaction.

\section{RESULTS AND DISCUSSION}

A total of five hydrodynamic and hydrostatic analyses were carried out for different aspect ratios. As a result, direct moments $\left(\mathrm{M}^{+}\right.$and $\left.\mathrm{M}^{-}\right)$per unit length at mid surface of an element on positive and negative faces about axis-1 and maximum top displacement $(\delta$-max) values were obtained, and are given in tables 2 and 3. Accordingly, few comparisons are made for the sake of brevity and shown in figures 5, 6, 7 and 8 . 


\begin{tabular}{|c|c|c|c|}
\hline \multirow{2}{*}{$\begin{array}{l}\text { Aspect } \\
\text { Ratio (A) }\end{array}$} & \multicolumn{3}{|c|}{$\begin{array}{c}\text { Hydrodynamic Analysis } \\
\text { Outputs }\end{array}$} \\
\hline & $M^{+} H D$ & $M_{H D}^{-}$ & $\delta-\max H D$ \\
\hline 1 & 60 & 45 & 3.19 \\
\hline 0.75 & 105 & 70 & 5.77 \\
\hline 0.6 & 160 & 90 & 8.07 \\
\hline 0.5 & 280 & 135 & 12.3 \\
\hline 0.43 & 515 & 322 & 45.04 \\
\hline
\end{tabular}

\begin{tabular}{|llll|}
\hline \multicolumn{4}{|l|}{ Table 3. Outputs of Hydrostatic Analysis. } \\
\hline $\begin{array}{l}\text { Aspect } \\
\text { Ratio (A) }\end{array}$ & $\begin{array}{l}\text { Hydrostatic } \\
\text { Outputs }\end{array}$ & Analysis \\
\cline { 2 - 4 } & $\boldsymbol{M}^{+} \boldsymbol{H S}^{-}$ & $\boldsymbol{M}_{\boldsymbol{H S}}$ & $\boldsymbol{\delta}$-max HS $^{\text {Hyy }}$ \\
\hline 1 & 48 & 22 & 1.3 \\
\hline 0.75 & 90 & 30 & 3.29 \\
\hline 0.6 & 125 & 33 & 7.22 \\
\hline 0.5 & 165 & 32 & 11.8 \\
\hline 0.43 & 195 & 28 & 16.4 \\
\hline
\end{tabular}

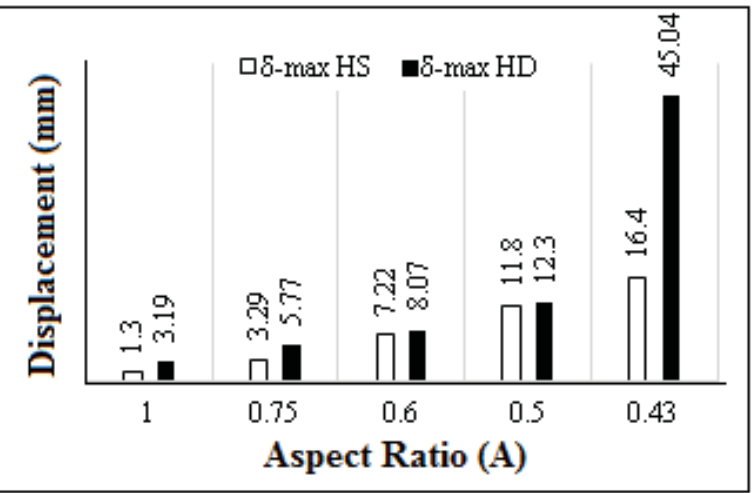

Figure 5. Top Displacement versus Aspect Ratio.

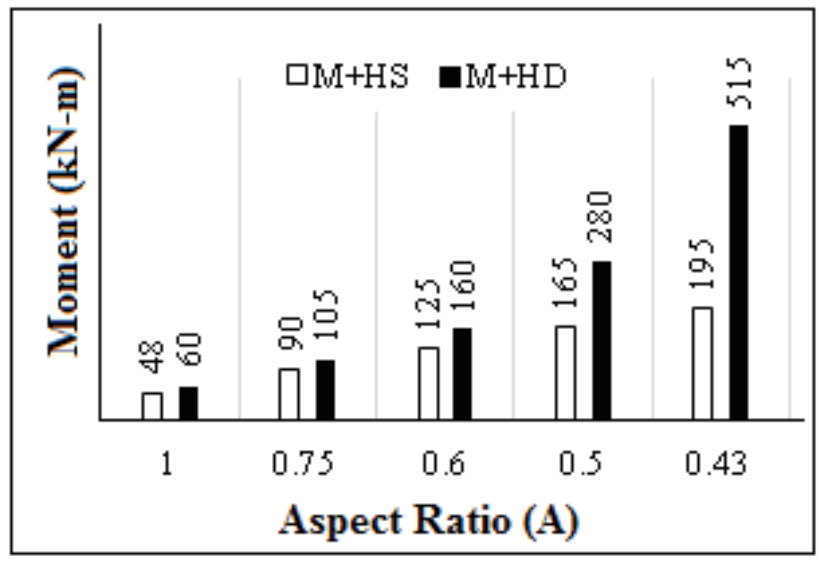

Figure 6. Moment on Positive Face versus Aspect Ratio.

The results show that, there is a smooth increase in the moment and displacement of both hydrostatic and hydrodynamic analysis with a decrease in aspect ratio (A). The displacement results are presented in figure 5. The moment and displacement due to hydrodynamic effects are significant as compared to hydrostatic. Moreover, it is observed that the hydrodynamic effect 
increases magnificently than the hydrostatic with the reduction of aspect ratio. This is due to the fact that the high flexibility associated with the convective mass which makes the structure susceptible for higher deformation.

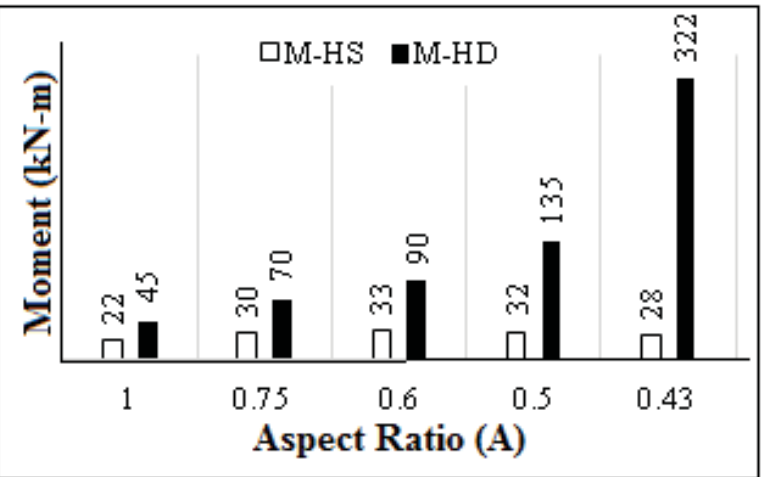

Figure 7. Moment on Negative Face versus Aspect Ratio.

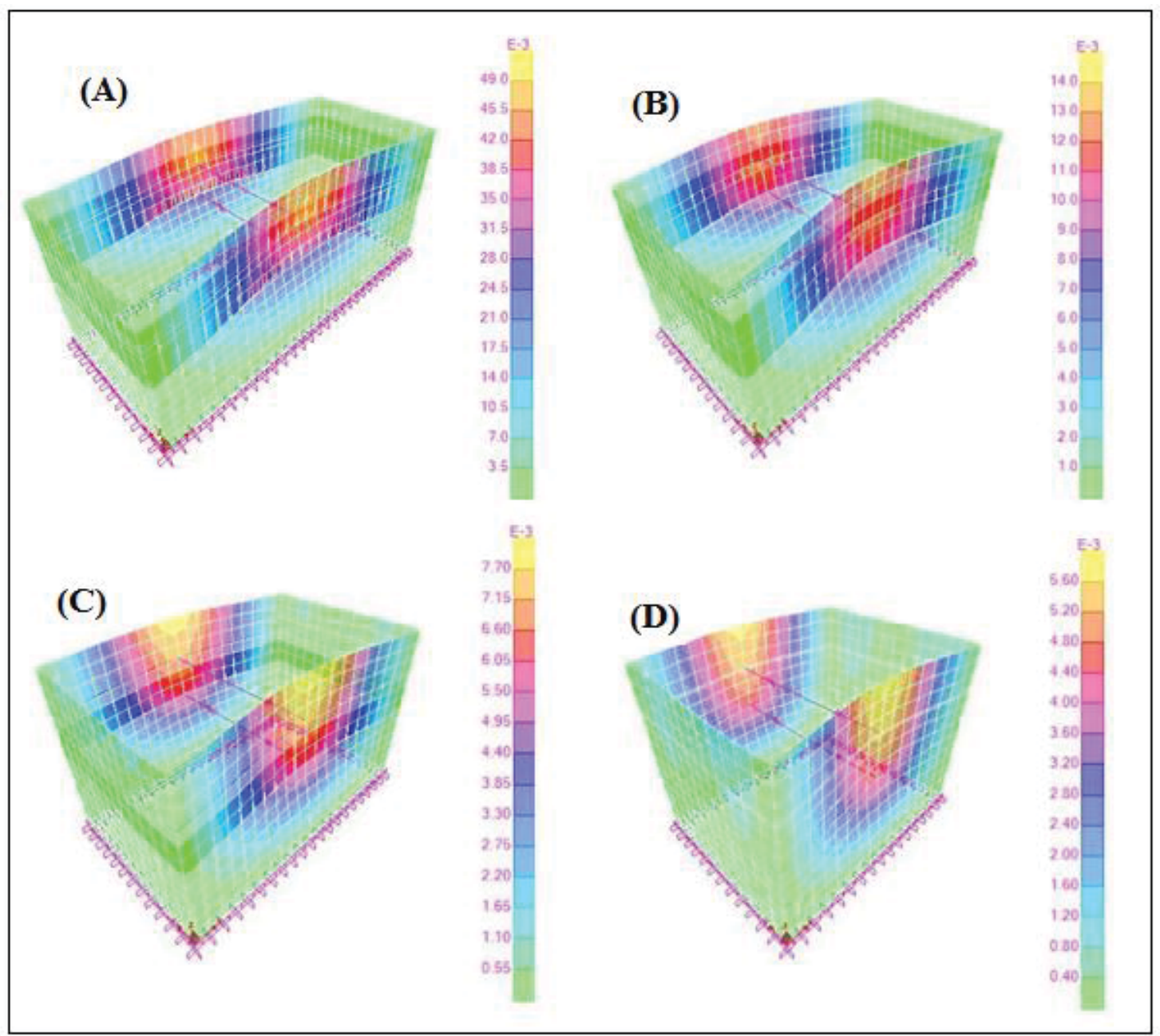

Figure 8. Deformed shape of tank (A) 14x6x6 size; (B) 12x6x6 size; (C) 10x6x6 size; (D) 8x6x6 size. 


\section{CONCLUSION}

This paper reviews the hydrostatic analysis and the hydrodynamic effect of earthquake induced forces on ground supported liquid storage tank structures. It is observed that, the results obtained based on dynamic analysis are critical for the design of such tank structures. In general the outcomes show that, there is a smooth increase in the moment and displacement of both hydrostatic and hydrodynamic analysis with a decrease in aspect ratio (A). The maximum hydrodynamic moment is observed to be $91.3 \%$ higher than the maximum hydrostatic moment. Similarly, the displacement obtained from hydrodynamic analysis is $63.58 \%$ more than the corresponding hydrostatic result. Thus a due consideration of hydrodynamic effects must be given in the design of liquid storage tank structures in seismic prone areas.

\section{ACKNOWLEDGEMENTS}

We are appreciative to the reviewers for their stimulating and constructive comments. Their remarks were supportive in producing quality paper that meets the standards. We are also thankful to this valued Journal for offering us this inordinate opportunity to publish our work.

\section{REFERENCE}

ACI350.3. 2001. Seismic design of liquid containing concrete structures. American Concrete Institute. Farmington Hill, MI, USA.

Chopra, A.K. 2006 Dynamics of Structures (Theory and Applications to Earthquake Engineering). $3^{\text {rd }}$ Edition, ISBN: 978-8131713297, Prentice-Hill.

Eurocode 8. 1998. Design provisions for earthquake resistance of structures, Part 1- General rules and Part 4 - Silos, tanks and pipelines. European Committee for Standardization, Brussels.

Helou, S.H. 2014. Seismic Induced Forces on Rigid Water Storage Tanks. Asian Journal of Engineering and Technology, 2(4): 2321-2462.

Housner, G.W. 1963. The dynamic behavior of water tanks. Bulletin of Seismological Society of America, 53(2): 381-387.

Jaiswal, O.R \& Jain, S.K. 2005a. Modified Proposed provisions for aseismic design of liquid storage tanks: Part I - codal provisions. Journal of Structural Engineering, 32(3): 195206. 
Jaiswal, O.R \& Jain, S.K. 2005b. Modified Proposed provisions for aseismic design of liquid storage tanks: Part II - Commentary and Examples. Journal of Structural Engineering, 32(4): 297-310. 